\title{
Prescripción social y confianza en adultos mayores: Una mirada en el contexto de COVID-19
}

$\mathrm{DOI}$

https://doi.org/10.35434/rcmhnaaa.2021.14Sup1.1176

\section{RESUMEN}

Introducción: El presente artículo tiene como objetivo realizar una revisión sobre prescripción social y confianza en torno al despliegue de las prestaciones sociales en los Centros del Adulto Mayor (CAM) del Seguro Social de Salud (EsSalud) durante la emergencia sanitaria por COVID-19. EsSalud en sus 126 CAM brinda prestaciones sociales a personas adultas mayores, esto es, actividades para el uso del tiempo libre que están ligadas a prescripción social; que al decretarse la emergencia sanitaria viraron a la modalidad virtual. La experiencia frente a la pandemia señala que la confianza en las instituciones varía en torno a las decisiones tomadas; ayudando a mantenerla, medidas como transparencia, colaboración entre interesados, entre otras; aspectos de interés si se desea que la población siga pautas sanitarias brindadas. En ese sentido, planteamos que las actividades de prescripción social pueden contribuir a la mejora de la confianza, debiendo realizarse investigación vinculante para la toma de decisiones.

Palabras Clave: Confianza; prescripción social; adultos mayores; Prestación de Atención de Salud; COVID-19; Perú (Fuente: DeCSBIREME).

\section{ABSTRACT}

Background: This article aims to carry out a review on social prescription and trust regarding the deployment of social benefits in the Elderly Centers (CAM) of the Social Health Security (EsSalud) during the health emergency due to COVID-19. EsSalud in its 126 CAM provides social benefits to older adults, that is, activities for the use of free time that are linked to social prescription; that when the sanitary emergency was decreed they turned to the virtual modality. Experience in the face of the pandemic indicates that trust in institutions varies around the decisions made; helping to maintain it, measures such as transparency, collaboration between stakeholders, among others; Aspects of interest if you want the population to follow the health guidelines provided. In this sense, we propose that social prescription activities can contribute to the improvement of trust, and must carry out binding research for decision-making.

Keywords: Trust; Social prescription; Aged; Delivery of Health Care ; COVID-19; Peru.(Source: DeCS-BIREME).
Social prescription and trust in older adults: A view in COVID.19 context

Zoila Romero-Albino ${ }^{1, a}$, Luis Ortigueria-Sánchez ${ }^{2, b}$

FILIACIÓN

1. Docente Facultad de Medicina Humana, Universidad Peruana de Ciencias Aplicadas, Lima, Perú.

2. Docente Departamento Académico de Administración, Facultad de Ciencias Empresariales, Universidad del Pacífico, Lima, Perú.

a. Magister en Project Management - ESAN, Magister en Geriatría y Gerontología - UPCH

b. Doctor en Administración de Empresas y Comercialización e Investigación de Mercados (Marketing), Universidad de Sevilla, España.

ORCID

1. Zoila Romero-Albino / 0000-0003-2519-2945

2. Luis Ortigueria-Sánchez / 0000-0002-0376-0166

\section{CORRESPONDENCIA}

Zoila Romero Albino

EMAIL

zoilara80@hotmail.com

\section{CONFLICTOS DE INTERÉS}

Los autores niegan conflictos de interés.

\section{FINANCIAMIENTO}

Autofinanciamiento.

\section{REVISION DE PARES}

Recibido: $12 / 06 / 2021$

Aceptado: 30/08/2021

\section{COMO CITAR}

Romero-Albino, Z., \& Ortigueria-Sánchez, L. Prescripción social y confianza en adultos mayores: Una mirada en el contexto de COVID-19. Revista Del Cuerpo Médico Hospital Nacional Almanzor Aguinaga Asenjo, 2021, 14(Sup1), 62 - 69. https://doi.org/10.35434/rcmhnaaa.2021.14Sup1. 1176

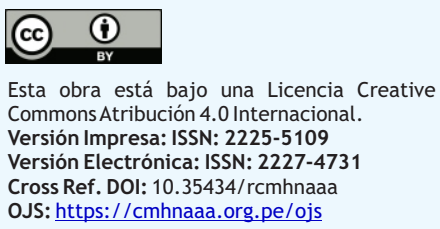




\section{INTRODUCCIÓN}

En nuestro país actualmente la población adulta mayor alcanza el $13 \%$ del porcentaje a nivel nacional de acuerdo a lo que señala el Instituto Nacional de Estadística e Informática $(\mathrm{INEI})^{(1)}$, previéndose su incremento en los próximos años. Esta situación nos plantea la necesidad de reestructurar los servicios, de tal forma que atiendan las necesidades y expectativas de esta población; y si bien, tenemos una brecha de tiempo a nuestro favor, se requieren decisiones audaces, concretas e innovadoras a nivel de Gobierno y de las instituciones públicas y privadas que en un lapso de tiempo perentorio nos permitan estar preparados para contener la demanda que significa el incremento de esta población.

Al haberse suscitado la pandemia generada por COVID-19, a nivel mundial los Gobiernos e instituciones públicas y privadas se vieron en la necesidad de replantear la forma como proveían sus servicios, sobretodo en el caso de los que atañen al área sanitaria, dado que estaban relacionados a contener la pandemia y mantener el estado de salud de la población. Este hecho también se observó en nuestro país, donde se declaró aislamiento social obligatorio y Estado de Emergencia Sanitaria, este último mantenido desde marzo del año 2020 a la fecha.

En el caso de los servicios que brindan cuidados a las personas adultas mayores en el Seguro Social de Salud (EsSalud), se cuenta con las Instituciones Prestadoras de Servicios de Salud (IPRESS) del lado de prestación sanitaria y los Centros del Adulto Mayor (CAM) del lado de la prestación social ${ }^{(2)}$. Estos últimos también se vieron en la obligación de replantear la provisión de sus servicios, hecho que en el caso de los CAM se realizó a través del uso de tecnología de información y comunicación (TIC), trasladando la prestación del servicio del modo presencial al modo virtual a través de una estrategia que se denominó CAM en CASA.

Las prestaciones sociales que brinda EsSalud desde hace más de 30 años, se configuran en una Cartera de Servicios que incide sobre la mejora de la capacidad funcional, mental y social de las personas adultas mayores autovalentes ${ }^{(2)}$; estos servicios que atañen al uso del tiempo libre de las personas adultas mayores se desarrollan dentro de los CAM, los mismos que dada su configuración actúan como "activos" para el despliegue de prescripción social en aras de la mejora del bienestar de esta población, de lo cual ahondaremos más adelante; no obstante, es necesario señalar que este modelo de prescripción social tiene sus bases en la concepción de salud desde el punto de vista salutogénico ${ }^{(3,4)}$, y que existe evidencia de su utilidad en el proceso de salud desde varias aristas, como es, la mejora de la confianza, autoestima, salud física, mental, entre otros.

Esta prestación que realiza EsSalud y que pasó al modo virtual a través del uso de las TIC, en un contexto tan complejo como el peruano donde existen brechas de desigualdad bastante marcadas, dilucidó que existiera cierta problemática en su implementación, que estuvo ligado al uso de las TICs, el acceso a internet, aspectos idiosincráticos de la población y de los mismos trabajadores, alfabetización digital, comunicación efectiva, entre otros problemas, lo cual también han sido observado en otros lugares del mundo. Este "salto" al modo virtual, que probablemente hubiera sido factible en años venideros, se convirtió en una realidad que arrasó los esquemas de trabajo a todo nivel, desnudando a la vez, la precariedad del sistema público.

Pero, ¿de qué manera influyen estas decisiones de Gobierno o de las instituciones?, ¿tienen implicancias en la confianza hacia las instituciones?, ¿es la confianza un factor clave para que los ciudadanos cumplan medidas impuestas por los Gobiernos? Para responder a estas preguntas se resalta el valor de la confianza, que denota la evaluación sobre el desempeño que tienen las instituciones o el Gobierno; evaluar los aspectos que la construyen y que la mantienen serán sustanciales para forjar un compromiso público para el bienestar común.

Al virtualizarse las prestaciones sociales realizadas en EsSalud en un contexto complejo como es el de la pandemia, un aspecto potencial que resaltamos, es la generación de confianza en la institución ${ }^{(5,6)}$; que desde el lado del usuario, se torna en una oportunidad para permanecer en sus hogares evitando el contagio, continuidad en sus actividades y sentimiento de protección por parte de la institución; y desde el lado de la gestión pública, vista en una oportunidad para incrementar la oferta de servicios, innovar y lograr un mayor alcance hacia población que no accedía al servicio.

En ese sentido, a través de la presente investigación brindamos aspectos teóricos sobre prescripción social, confianza, despliegue de la prestación social dirigida a personas adultas mayores en EsSalud, pero también planteamos la necesidad de mayor investigación que dilucide la relación existente entre el desarrollo de actividades de prescripción social sobre la confianza en un contexto de emergencia.

\section{CONTENIDO}

\section{Prescripción social}

La recomendación de activos o prescripción social, se entiende como el proceso por el cual un profesional de la salud recomienda a una persona "recursos o activos" de la propia comunidad donde reside en beneficio de su salud y bienestar integral. Estos activos, pueden ser asociaciones juveniles, de personas adultas mayores, vecinales, deportivas, culturales; servicios voluntarios; grupos de caminata y lectura; plazas o parques que sirven de espacio para el encuentro e interacción y que se encuentran dentro de la comunidad ${ }^{(3,4,7)}$

El modelo de prescripción social (figura 1 ) está relacionado con el entendimiento que la concepción de salud no está meramente relacionado a un modelo patogénico, sino a un modelo salutogénico, por el cual toda persona, comunidad u organización tiene activos en salud a través de los cuales puede sobrellevar los problemas; esto es, en el entendimiento de que la salud de una persona está determinada por una amplia gama de cuestiones sociales, económicas, ambientales que se encuentran en el entorno proximal y remoto de la persona; que si se manejan adecuadamente permiten el apoyo holístico, que se adapta a las necesidades del individuo ${ }^{(3,4)}$. 
La prescripción social puede realizarla cualquier profesional de la salud y es particularmente útil para bordar necesidades sociosanitarias, para lo cual previamente se debe realizar un mapeo de activos que se disponen en la comunidad; así como, la creación de vías de derivación que permita la mejora de su salud y bienestar ${ }^{(3,4)}$.

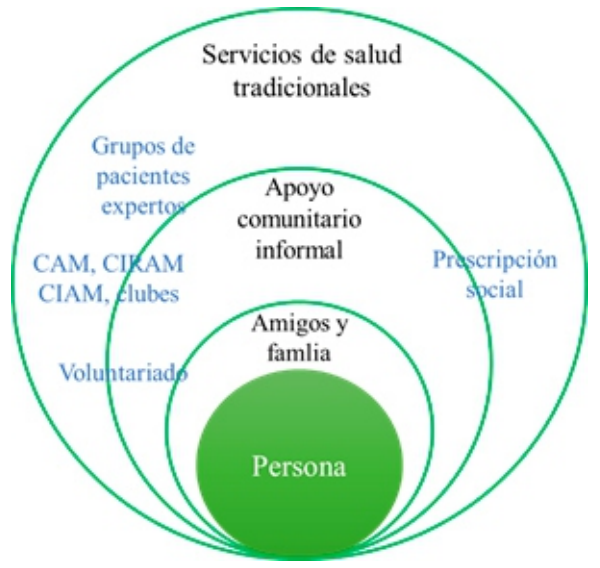

Figura 1.

Relación entre enfoque centrado en la persona y soporte adecuado. Elaboración propia, adaptada de Boydell K. The Art of Social Prescription. (Centro del Adulto Mayor: CAM, Círculo del Adulto Mayor CIRAM, Centro Integral del Adulto Mayor - CIAM).

La forma de derivación hacia los activos en la comunidad puede realizarse de diversas maneras, que pueden caracterizarse en 4 tipos, asesoramiento o consejo de activos comunitarios, recomendación básica de un activo específico, recomendación de activos facilitada y recomendación de activos avanzada, estas dos últimas requieren de un facilitador y canales de comunicación a diferencia de los primeros $^{(3)}$.

La prescripción social es un tipo de acción comunitaria en salud; ambas se inician con el desarrollo de alianzas, que en el caso de la prescripción social se continua con la identificación y priorización de los activos que a nivel de la comunidad se van a recomendar; posteriormente, se realiza la búsqueda de evidencia respecto a los activos, relacionada a sus implicancias en la promoción de la salud y sistema de acogida, para finalmente pasar a la acción, monitoreo y evaluación. Es pertinente señalar que tanto la acción comunitaria en salud como la prescripción social, facilitan la reducción de inequidades y el empoderamiento del individuo en la comunidad ${ }^{(8)}$

El proceso por el cual el individuo logra bienestar, se ha podido señalar a través de pasos, que inician con el soporte del profesional y la derivación hacia los activos de la comunidad, donde la persona participa de ciertas actividades y conecta socialmente; seguido del aprendizaje, donde la persona se relaciona y adquiere nuevas destrezas o habilidades; continúa con la percepción de cambio o la concientización de que es posible una mejora futura y finalmente el desarrollo de una perspectiva positiva de la vida $^{(9)}$.

Dentro de las actividades de prescripción social usadas se puede encontrar la prescripción de arte (danza, música, poesía), biblioterapia, alfabetización, actividad física, ecoterapia, iniciativas de vida saludable en la comunidad, entre otras.

\section{Utilidad de la prescripción social}

La evidencia sobre prescripción social se refleja en resultados positivos para la salud y el bienestar, con el incremento de la autoestima, confianza, sentido de la vida, empoderamiento, incremento de redes sociales, sociabilidad, mejora de la salud física, bienestar emocional; disminuyendo el aislamiento social y los niveles de depresión, estrés y ansiedad; así como su utilidad en el control de enfermedades crónicas, a través de la mejora en estilos de vida ${ }^{(9,10)}$.

En otra experiencia el servicio de prescripción social ha permitido que las personas desarrollen una visión más optimista de la vida, dado que pueden participar de diversas actividades en la comunidad, que son posibles gracias a la participación de un facilitador o coordinador de la prescripción que activan y ejercen un impacto inicial ${ }^{(11)}$; no obstante, estas intervenciones no han demostrado que efectivamente la persona haga menos uso de los servicios sanitarios.

La prescripción social a través del uso de intervenciones artísticas tiene beneficios en la reducción de síntomas físicos y mejora de la salud emocional como se ha evidenciado en una revisión de 20 artículos realizada por Jensen y Bonde en el año $2018^{(12)}$.

No obstante, a esta evidencia, en una revisión sistemática realizada en Reino Unido, se concluyó que la evidencia de los programas de prescripción social era insuficiente. En esta revisión se tomó como variable resultado la mejora de salud física o emocional y la frecuencia con la que acuden al consultorio de atención primaria ${ }^{(13)}$.

Uso del tiempo libre y prescripción social para personas adultas mayores

La prescripción social que promueve un envejecimiento activo puede actuar sobre 3 categorías funcionales, como son, la mejora de la salud y capacidad física, mejora de la cognición y mejora de la salud mental y bienestar emocional, y las mismas pueden desarrollarse teniendo en cuenta características, necesidades y expectativas del individuo; sus estilos de vida y entorno en el que se desenvuelve ${ }^{(14)}$.

Mirando más de cerca las actividades que realizan las personas adultas mayores en su tiempo libre (actividades de ocio), clasificadas en tres tipos, como ocio activo, ocio social (para interacción) y tiempo libre; estas pueden utilizarse en el marco de prescripción social, dado su potencial como activos dentro de la comunidad.

Sobre la eficacia de estas intervenciones en el bienestar emocional de las personas adultas mayores, en un estudio realizado en Korea en el año 2015, se encontró que eran las actividades de ocio activas (deportes, yoga, etc.) las que contribuían en mayor medida al disfrute del envejecimiento, brindándoles mayor satisfacción y felicidad; siendo que, forma general, todas las actividades de ocio potenciaban la salud emocional ${ }^{(15)}$; esto concuerda a lo encontrado en un estudio realizado en Tehran donde se encontró que existía 
Cuadro 1. Mejoras potenciales que pueden lograrse a través de actividades de prescripción social en personas adultas mayores. Elaboración propia.

Bienestar emocional: satisfacción, felicidad, integración laboral.

\begin{tabular}{ll}
\hline Lectura, escritura y uso de internet ${ }^{(17,19,24)}$ & $\begin{array}{l}\text { Efectos positivos en la depresión y en la calidad de vida. } \\
\text { Mejora cognitiva: memoria, lenguaje, atención. }\end{array}$ \\
\hline${\text { Actividades sociales y de comunicación }{ }^{(17)}}^{\text {Efectos positivos en la depresión y en la calidad de vida }}$ \\
\hline Aeróbicos, ejercicios de resistencia $^{(20)}$ & Mejora cognitiva. \\
\hline Turismo, visitas a parques, museos, otros ${ }^{(17,21) .}$ danza, teatro, música, artes visuales, narraciones otros ${ }^{(22)}$ & Bienestar subjetivo, emociones positivas. \\
\hline Jugar cartas, escuchar música, otras relacionadas ${ }^{(23)}$ & Mejora cognitiva, atención compleja, aprendizaje, memoria. \\
\hline Actividades de jardinería & Prevención de soledad o aislamiento. \\
\hline
\end{tabular}

relación significativa entre la participación en actividades de ocio y la salud mental, pero mediadas a través de actividades físicas ${ }^{(16)}$. Además, en un estudio realizado en Korea, se encontró que las actividades de ocio (sociales, emocionales, activas, entre otras), tenían efectos positivos en la depresión y en la calidad de vida de esta población ${ }^{(17)}$. Sobre el impacto en el envejecimiento de las personas adultas mayores a través de actividades de ocio, en un estudio realizado en Japón se encontró que la participación en estas actividades ayuda a esta población a mantener el bienestar integral, esto es, la salud mental, cognitiva y física ${ }^{(18)}$.

Las actividades de ocio también influyen en la cognición, es así que la participación reducida en estas actividades, ha demostrado ser un factor predisponente a demencia tal como se demostró en un estudio realizado en Beijing a través de pruebas neuropsicológicas y resonancia magnética ${ }^{(19)}$, que concuerda con lo encontrado en una revisión sistemática donde se encontró que los aeróbicos y la combinación de entrenamiento aeróbico / ejercicios de resistencia, mejoraban de manera más consistente la función cognitiva $^{(20)}$.

Al referirnos a actividades de ocio específicas y su relación con la mejora de diversos procesos mentales, cognitivos o funcionales de las personas adultas mayores, podemos señalar al turismo o visitas a parques, museos otros, que ha demostrado tener relación con la satisfacción de necesidades psicológicas (competencia, afinidad y autonomía), dado que brinda experiencias de emociones positivas y bienestar subjetivo $^{(21)}$. En el caso de las artes (danza, teatro, música, entre otros), de acuerdo a una revisión sistemática, ha demostrado un efecto positivo en personas adultas mayores con deterioro cognitivo leve, mejorando aspectos como cognición global, atención compleja, aprendizaje y memoria, entre otros ${ }^{(22)}$. Sobre las actividades de ocio como jugar las cartas, ver televisión o escuchar música y actividades sociales, han sido efectivas para prevenir la soledad o aislamiento ${ }^{(23)}$. Otras actividades de ocio, como el uso del internet en personas adultas mayores, ha demostrado contribuir en la reserva cognitiva permitiendo un envejecimiento con bienestar, existiendo diferencias por género, con mayor beneficio para los varones ${ }^{(24)}$; este dato es importante dado que en el marco de la pandemia por COVID19 su uso se ha incrementado para el despliegue de las actividades de ocio para personas adultas mayores en el caso de EsSalud. En el caso de actividades de jardinería, también se ha evidenciado su utilidad como actividad de prescripción social, teniendo evidencia de su utilidad en la mejora del bienestar emocional, mejora de patrones fisiológicos y reducción de aislamiento ${ }^{(25)}$.

\section{USO DE TECNOLOGÍA PARA DOTACIÓN DE SERVICIOS SANITARIOS Y SOCIALESA PERSONAS ADULTAS MAYORES}

El contexto de cuarentena en nuestro país, por el cual las personas adultas mayores han quedado recluidos en sus casas, ha hecho necesario repensar en nuevas e innovadoras formas de poder dotar los diversos servicios públicos, siendo el uso de las TICs, una de las formas que ha adquirido gran relevancia; sin embargo, para las personas adultas mayores podría constituirse en un reto no sólo por la brecha digital a la que se enfrentan, sino también ligado al acceso a internet y conectividad, entre otros problemas ${ }^{(26)}$, lo cual podría aumentar su aislamiento, o tener sentimientos de baja autoeficacia, ansiedad y hostilidad cuando no pueden usar las diversas tecnologías que se le presentan ${ }^{(27)}$.

El uso de esta tecnología ha sido más evidente en el campo de la atención sanitaria a través del uso cada vez más frecuente de diversas aplicaciones móviles para asistencia sanitaria a personas adultas mayores independientes ${ }^{(28)}$; que ha seguido la misma ruta marco en el contexto por COVID-19, con el uso de aplicativos, $\mathrm{y} / \mathrm{o}$ de la inteligencia artificial.

El uso de estas herramientas para la prestación sanitaria a personas adultas mayores, ha dado lugar por un lado, a que disminuya la exposición al no tener que acudir a espacios con mayor congestión de personas, disminuya tiempos de viaje, reduzca tiempos de espera y permita un acceso más rápido; pero también ha generado la necesidad de regularizar el marco normativo, definir los tipos de equipos más adecuados para estas personas, definir responsabilidades y roles, y definir qué tipos de atenciones son apropiadas realizar a 
través de estas herramientas ${ }^{(29)}$.

Sobre la experiencia en el uso de la tecnología, en un estudio, se encontró que el $32 \%$ de las personas adultas mayores no se sentían preparadas para el uso de telemedicina; no obstante, el uso del teléfono podría mejorar el acceso sobre todo en aquellos con alguna discapacidad, por lo que se requieren políticas que sepan reconocer la brecha digital de los usuarios y plantear mejoras de acuerdo al contexto ${ }^{(30)}$.

Respecto al impacto, en un estudio se evaluó su connotación respecto al deterioro cognitivo a través del uso de medios como televisión o teléfono, encontrándose que el uso de estos medios, demostraron potencial para la estimulación cognitiva ${ }^{(31)}$. Así mismo, en otro estudio se encontró que existían algunos factores que podían predecir si la adopción de la telemedicina podría ser exitosa, encontrándose que factores tales como utilidad percibida, expectativa del esfuerzo, influencia social, seguridad percibida, ansiedad informática, condiciones facilitadoras, entre otros, resultaban importantes en la planificación estratégica para la adopción de estos en personas adultas mayores ${ }^{(32)}$.

Para el caso puntual de prescripción social realizada a través de TICs, a nivel internacional existen informes que señalan su utilidad en niños y personas jóvenes ${ }^{(33)}$, resaltándose su valor disruptivo si se superan barreras estratégicas, tácticas y operativas que permitan la adaptación al usuario y sean de fácil manejo. En el caso de Perú, no existen estudios que evidencien el uso de la prescripción social en personas adultas mayores a través de las TICs.

\section{CONFIANZA EN LAS INSTITUCIONES / GOBIERNO}

La problemática mundial en torno a COVID-19 ha generado discusión sobre las decisiones que han tomado los diversos gobiernos en torno a los servicios públicos; es así que, una aproximación que puede valorar si estas decisiones fueron las mejores, está referida a medir la confianza generada en el ciudadano sobre el Gobierno y/o instituciones públicas a raíz de las medidas adoptadas.

Sobre esta temática, Aitalieva y Park, señalan que el "éxito de los gobiernos en la introducción de políticas de salud dependerá en gran medida tanto de las percepciones de sus ciudadanos como del papel apropiado de los gobiernos y de la capacidad de respuesta de los gobiernos a la voluntad popular"; entendiéndose que la confianza política se define como la evaluación que hacen los ciudadanos sobre el desempeño de los gobiernos y que está relacionado con sus expectativas $^{(34)}$.

La construcción de confianza está relacionada con varios factores, entre los que se señala, la confianza en las habilidades y capacidades de las personas, fe en el poder de los sistemas de gobernanza, beneficios colaterales, transparencia y experiencias positivas propias o ajenas ${ }^{(35)}$. Esta construcción de la confianza contribuye al mantenimiento de la misma a través de una comunicación regular, crédito y poder, empatía, flexibilidad, alcance de las metas, transparencia, desarrollo de protocolos, proactividad a la credibilidad, la persona en el centro, colaboración entre los interesados, consistencia, educación de todos los interesados, construcción de la reputación y cumplimiento de promesas $^{(35)}$, siendo un mediador importante, la participación ciudadana.

Tanto la construcción, como el mantenimiento de la confianza, están expuestos a factores que la amenazan, por tanto, es imprescindible un monitoreo continuo que evite la pérdida de la misma ${ }^{(35)}$, y que en contexto como el de la pandemia generada por COVID-19, permitan que la población pueda seguir los consejos brindados por el estado y así mantenga su salud en niveles óptimos; siendo importante entonces, la medida de la calidad a través de indicadores de desempeño subjetivos y objetivos.

Sobre la confianza en torno a la pandemia por COVID-19, en un estudio realizado en Tailandia entre febrero y marzo del año 2020 donde se evaluó la confianza pública, se encontró que ésta, estaba mediada por la confianza profesional o en los expertos, lo que se relacionaba a cumplimiento de medidas sanitarias, tales como uso de mascarilla, lavado de manos, evitar zonas de riesgo y uso de utensilios compartido con otras personas ${ }^{(5)}$. En otro estudio realizado en 177 países donde se utilizó modelos regresión lineal, se estudió como influía la confianza pública en el comportamiento de las personas, y se hizo una distinción de acuerdo al régimen político, encontrándose que en gobiernos donde las políticas eran más restrictivas, hubo un mayor cumplimiento, esto además estaba ligado al rigor en que se determinaban estas políticas tanto en países con gobiernos autoritarios como democráticos ${ }^{(6)}$

Sobre la comunicación, en un estudio realizado en 7 países europeos concluyó que era necesario que los gobiernos adoptaran estrategias de comunicación de tal manera que los ciudadanos fueran capaces de seguir las medidas impuestas por periodos largos, lo que está relacionado con la confianza que tienen en el gobierno ${ }^{(36)}$.

Es de señalar que existe evidencia de que la confianza como parte del constructo capital social y las desigualdades económicas también influyen en aspectos como mortalidad, tal como lo señala el estudio de Elgar et al, realizado en 84 países donde se encontró que la mortalidad por COVID-19 se relacionaba positivamente con la desigualdad económica y la pertenencia a grupos, y negativamente con la participación cívica y la confianza en las instituciones públicas, señalando, que esto se podía interpretar a través de teorías del capital social que diferencian las relaciones sociales horizontales de las verticales ${ }^{(37)}$, de lo cual todavía se requieren más estudios.

Para nuestro entorno no se ha encontrado estudios que relacionen la confianza en el desarrollo de políticas públicas en el contexto de pandemia, ni tampoco si esta es susceptible a cambios respecto a la variación de los servicios sociosanitarios.

\section{Confianza y prescripción social}

Hasta el momento, no se ha encontrado estudios que relacionen la confianza con el desarrollo de actividades de prescripción social ni a nivel internacional, ni en nuestro entorno, lo cual revela la necesidad de una mayor investigación en este campo, sobre todo en un contexto pluricultural y de desgobernanza como es el peruano.

Sin embargo; es necesario señalar que, dentro de los 
determinantes de bienestar subjetivo que la OMS señala y que explican la gran variabilidad entre los países se encuentran, el sentido de libertad, la generosidad, tener a alguien con quien contar y la confianza; esta última, dado que incrementa la resiliencia y permite la satisfacción con la $\operatorname{vida}^{(38)}$. En ese sentido, las políticas sanitarias tendrían que mirar hacia el logro de la misma a través de actividades diversas, que no sólo incluyan las sanitarias en forma per se, sino las relacionadas al contexto social, y la interacción que ejercen en el individuo para lograr así mayor bienestar.

Entonces, siendo las actividades de prescripción social desplegadas dentro de la comunidad acciones que contribuyen a mejorar la cognición, bienestar emocional y la memoria, entre otros aspectos ya señalados ${ }^{(9,10,12)}$, su potencialidad para influir sobre la confianza ciudadana podría ser alta, de ello la relevancia de estudios de esta índole.

Alguna evidencia que puede señalarse en este marco, es la relacionada a capital social con énfasis en apoyo, confianza y cohesión, donde en un estudio realizado en 7,714 personas adultas mayores se encontró que los que vivían solos reportaban menos confianza y cohesión social, haciéndolos más propensos al uso de los servicios sanitarios; no así, las personas adultas mayores con apoyo social o que participaban de actividades en su tiempo libre. En este estudio se sugirió la posibilidad de asociaciones bidireccionales entre capital social y salud en adultos mayores $^{(39)}$.

\section{PRESTACIONES SOCIALES EN ESSALUD}

EsSalud de acuerdo a su Ley de creación, brinda prestaciones sanitarias, sociales y económicas a población asegurada. En el caso de las prestaciones sociales estas vienen desplegándose en los CAM desde hace más de 30 años y tienen como fin lograr en las personas adultos mayores un envejecimiento activo, productivo y saludable $e^{(2)}$. Cabe señalar que el servicio que se brinda en los CAM está dirigido a personas adultas mayores autovalentes, no considerando por tanto a las personas adultas mayores frágiles o dependientes.

A nivel nacional existen 126 CAM, 40 de los cuales se ubican en Lima ${ }^{(2)}$, los mismos que se dividen en 3 categorías (I, II y III), definidos de acuerdo a la cantidad de usuarios y de servicios que ofrecen, reconociéndose en cada una de las categorías funcionamientos diferentes. Los CAM han mantenido a lo largo de los años una forma de gestión interna sui géneris, son administrados por un profesional trabajador social o psicólogo, funcionan en locales propios o alquilados y cuentan con un equipo de coordinadores de actividades compuestos por adultos mayores elegidos por los propios usuarios.

Los servicios que se ofrecen en los CAM, están relacionados a actividades para el uso del tiempo libre, sistematizados en una "Cartera de Prestaciones Sociales para el Envejecimiento Activo", la misma que incide sobre la mejora de la capacidad funcional, mental y social de las personas adultas mayores ${ }^{(2)}$; sin embargo, esta dotación de servicios no es homogénea a nivel nacional, sino que está condicionada en la mayor parte de los casos por la capacidad de los ambientes que disponen, por las preferencias de las personas adultas mayores y por o la posibilidad de pago voluntario que realizan para poder solventar algunos talleres que son de su interés y que no son brindados por la institución.

A nivel nacional, se evidencia una carencia de estudios sobre los modelos o formas de gestión interna de los CAM, así como

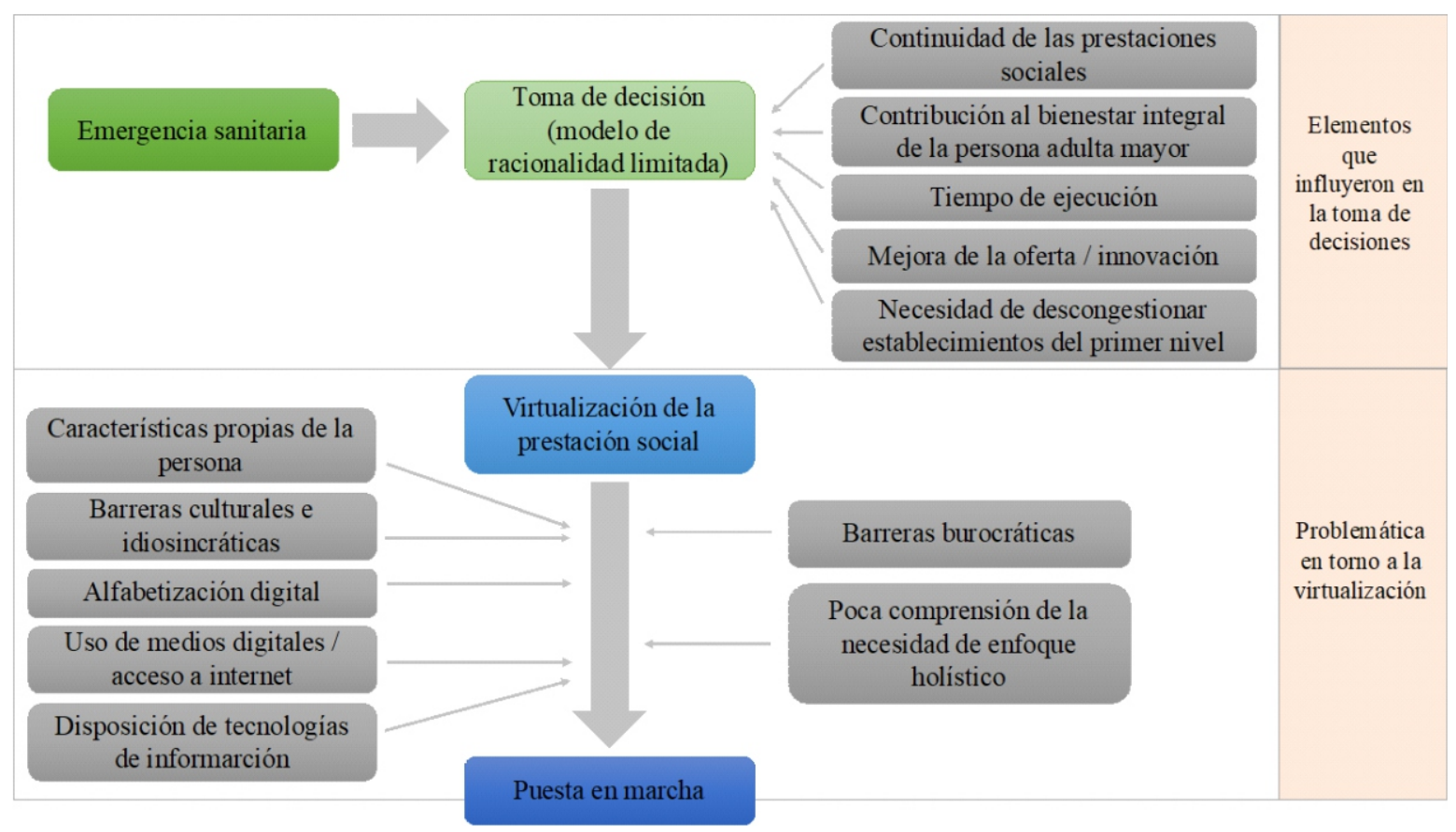

Figura 2.

Proceso para la puesta en marcha de virtualización de la prestación social. Elaboración propia 
de la cuantificación del impacto que provocan los servicios sobre la calidad de vida de las personas adultas mayores, sobre todo considerando que estás permitirán relentecer el proceso de disminución de la capacidad funcional y cognitiva de esta población; no obstante, la evidencia internacional señala la repercusión que estas actividades generan en la mejora del bienestar de esta población como hemos visto en acápites anteriores ${ }^{(15,16,18)}$

Las actividades desplegadas dentro de los CAM dentro de un espacio territorial, puede considerarse como un "activo" potencial para el desarrollo de prescripción social, que, articulado correctamente con el servicio sanitario, contribuiría en mayor medida a mejorar el bienestar integral de las personas adultas mayores que acoge.

Proceso de toma de decisiones en el marco de la emergencia sanitaria generada por COVID-19

En el contexto de emergencia sanitaria, en EsSalud se decidió la suspensión de los servicios presenciales en los CAM, determinándose el pase de las diversas actividades a la modalidad virtual tanto en su forma sincrónica como asincrónica, haciendo uso para ello de diversas plataformas como zoom, meet, youtube, entre otras. El proceso de decisión, concuerda con el Modelo de racionalidad limitada de Simon, en donde se trata de buscar un término medio entre el análisis del problema y la urgencia de ofrecer una solución rápida y aceptable, cabe señalar que en este modelo el ejercicio de la razón se encuentra supeditado al contexto social, político y cognitivo en el cual actúa el decisor ${ }^{(40)}$, y puede verse en la figura 2 .

La modalidad virtual conllevó diversos retos en los diferentes niveles de organización, que viraron desde la aprobación del marco normativo correspondiente, hasta el adiestramiento de las personas y/o familiares al uso de medios informáticos, superar problemas de conectividad y barreras burocráticas relacionadas a los procesos de contrataciones, lo cual puede observarse en la figura 2. Es de precisar que la figura señala el proceso para el caso de la población usuaria a los CAM, es decir personas adultas mayores autovalentes; no así de frágiles y dependientes, cuyos retos son distintos.

Cabe señalar, que la puesta en marcha de la prestación social al modo virtual, estuvo acompañada de un proceso de comunicación interna y externa.

El proceso de virtualización en un contexto complejo como es el de la pandemia generada por COVID-19 bien gestionado tiene el potencial para mejorar la confianza; dado que, que visto desde el usuario, se torna en una oportunidad para mantenerse en sus hogares evitando el contagio de la enfermedad, permite continuidad en el desarrollo de sus actividades que mejoran la interacción social, permite el acceso a medidas educativas de prevención de la enfermedad y promoción de la salud y genera un sentimiento de protección e interés por parte de la institución y el Estado, aspectos valiosos que ameritan mayor atención.

Sin embargo, la virtualidad también supone algunas desventajas, por ejemplo, no permite el contacto personal que en momentos de crisis emocional es importante; así mismo, es necesaria su adecuación para el caso de poblaciones quechua hablantes, comunidades selváticas, personas con discapacidad, entre otras.

Siendo entonces los CAM activos potenciales para el desarrollo de prescripción social, su contribución a la mejora del bienestar integral de las personas adultas mayores es invaluable y merece mayor investigación. Así mismo, en el caso de las actividades de prescripción social, por su naturaleza holística en el cuidado de la salud y desarrolladas bajo la modalidad virtual en un contexto de pandemia por COVID-19, se tornan en una apuesta disruptiva que puede mejorar la confianza del ciudadano. Evidentemente toda apuesta de cambio, conlleva retos, que en el caso de la gestión pública implica una mirada macro, tendente a romper barreras de toda índole, y que en el caso peruano se tornan complejas dadas las desigualdades, corrupción a todo nivel y desgobernanza; no obstante, miramos con confianza este tipo de acciones que fortalecen la atención primaria y permiten un envejecimiento activo, productivo y saludable.

\section{CONCLUSIONES}

La prescripción social mejora el bienestar de las personas, sobre todo si se articula desde la atención primaria; sin embargo, la implementación en nuestro país plantea desafíos relacionados con la falta de un enfoque estratégico, aceptación de parte de los decisores y profesionales de la salud, rotación del personal, recursos financieros, falta de enfoque integral, entre otros aspectos que es necesario evaluar de acuerdo al contexto donde se pretende desarrollar.

La confianza del ciudadano en las instituciones está ligada a diversos factores, tales como respuesta política, transparencia, desarrollo de protocolos, credibilidad, atención centrada en la persona, colaboración entre los interesados, construcción de la reputación, cumplimiento de promesas, comunicación eficaz, comportamiento protector, entre otros múltiples factores, que deben tenerse presente para el mantenimiento de la misma y desarrollo de respuestas eficaces por parte de los ciudadanos en contextos complejos como son los de emergencia sanitaria.

Los CAM de EsSalud se constituyen en un activo para la prescripción social, debiendo articular con las prestaciones sanitarias y con otros activos dentro de la comunidad que permitan a las personas adultas mayores disponer de diversos servicios para la mejora de su bienestar.

En EsSalud, el proceso de virtualización de las prestaciones sociales en el marco de la emergencia sanitaria ha implicado la toma de decisiones dentro de la organización y se constituye en una experiencia retadora para la gestión. Así mismo, el uso de las TICs abre la posibilidad de ampliar la oferta a personas adultas mayores frágiles y dependientes que actualmente no gozan de los beneficios de los servicios que ofrecen los CAM.

Se plantea que las actividades de prescripción social en personas adultas mayores, potencialmente podrían mejorar la confianza del ciudadano, no existiendo estudios al respecto que vinculen ambas variables; sin embargo, consideramos su potencialidad y por ende insistimos en el desarrollo de investigaciones vinculantes que finalmente contribuirán a que puedan implementarse en las diversas 
organizaciones sanitarias.

\section{REFERENCIAS BIBLIOGRÁFICAS}

1. Instituto nacional de estadística e informática. Informe Técnico: Situación de la población adulto mayor. Lima: INEI;2021 [citado el 27 de Julio del 2021]. Disponible en: https: / / bit.ly/3u3kpb3

2. EsSalud. Plan Estratégico Institucional 2020-2024. Lima: EsSalud. 2020.

3. Calderón-Larrañagaa S, Braddick F. De la prescripción social a la recomendación de activos: ¿qué funciona, para quién, en qué contexto y cómo?. FMC. 2021;28(Extraordin 1):3-11. DOI: 10.1016/j.fmc.2021.02.002

4. Boydell K. The Art of Social Prescription. En: Rhodes P, editor. Beyond the Psychology Industry. Springer. Suiza; 2020. p.128-152.

5. Saechang O, Yu J, Li Y. Public Trust and Policy Compliance during the COVID-19 Pandemic: The Role of Professional Trust. Healthcare. 2021;9(2):151. doi: 10.3390 / healthcare9020151.

6. Pak A, McBryde E, Adegboye O. Does High Public Trust Amplify Compliance with Stringent COVID-19 Government Health Guidelines? A Multi-country Analysis Using Data from 102,627 Individuals. Risk Management and Healthcare Policy. 2021;14:293-302. doi: 10.2147 / RMHP.S278774.

7. Clements-Cortés A, Yip J. Social Prescribing for an Aging Population. Activities, Adaptation \& Aging. 2020;44(4): 327-340. DOI: 10.1080/01924788.2019.1692467

8. Poblet C, González-Vianab A. Herramientas para una orientación comunitaria de la atención primaria: el mejor sustrato para la prescripción social. FMC. 2021;28(3):21-32. Doi: 10.1016/j.fmc.2021.02.004

9. Payne K, Walton E, Burton C. Steps to benefit from social prescription: a qualitative interview study. British Journal of General Practice. 2020;70(690):e36-e44. doi: 10.3399 / bjgp19X706865. Imprimir 2020 Ene.

10. Costa A, Sousa CJ, Seabra P.R.C, Virgolino A, Santos O, Lopes J, Henriques A, Nogueira P, Alarcão V. Effectiveness of Social Prescribing Programs in the Primary Health-Care Context: A Systematic Literature Review. Sustainability. 2021;13:2731. Doi: $10.3390 /$ su13052731.

11. Bertotti M, Frostick C, Hutt P. Sohanpal R, Carnes D. A realist evaluation of social prescribing: An exploration into the context and mechanisms underpinning a pathway linking primary care with the voluntary sector. Primary Health Care Research \& Development. 2018;19(3): 232-245. Doi: 10.1017 / S1463423617000706.

12. Jensen $A$, Bonde $L$. The use of arts interventions for mental health and well-being in health settings. Perspectives in Public Health. 2018; 138(4): 32-45. doi: 10.1177 / 1757913918772602.

13. Public Health England. Effectiveness of social prescribing - An evidence synthesis. London: PHE publications; 2019.

14. Gray M, Adamo G, Pitini E, Jani A. Precision social prescriptions to promote active ageing in older people. Journal of the Royal Society of Medicine. 2020;113(4):143-147. doi: 10.1177 / 0141076819865888.

15. Bum $\mathrm{CH}$, Johnson J, Choi Ch. Healthy Aging and Happiness in the Korean Elderly Based upon Leisure Activity Type. Iran J Public Health. 2020;49(3):454-462. Doi: 10.18502/ijph.v49i3.3141

16. Hosseine S, Tajvar M, Dehghan N, Rahimi A, Akbari A, Siar A, et al. Leisure time activities and mental health among iran older people: a population-based study in Tehran. Int J Physiother. 2019;6(3):75-81. Doi: 10.15621/ijphy/2019/v6i3/183875

17. Jeong EH, Park JH. The Relationship Among Leisure Activities, Depression and Quality of Life in Community-Dwelling Elderly Koreans. Gerontology \& Geriatric Medicine. 2020;6:1-7. doi: 10.1177 / 2333721420923449.

18. Sala G, Jopp D, Gobet F, Ogawa M, Ishioka Y, Masui Y, et al. The impact of leisure activities on older adults'cognitive function, physical function, and mental health. PLoS ONE. 2019;14(11):e0225006. DOI: 10.1371 / journal.pone.0225006

19. Chen Y, Lv Ch, Li X, Zhang J, Chen K, Liu Z, et al. The positive impacts of early-life education on cognition, leisure activity, and brain structure in healthy aging. Aging. 2019;11(14):4923-42. doi: 10.18632/aging. 102088 .

20. Sprague B, Freed S, Webb C, Phillips C, Hyun J, Ross L. The impact of behavioral interventions on cognitive function in healthy older adults: A systematic review. Ageing Research Reviews. 2020;52:32-52. doi: 10.1016 / j.arr.2019.04.002.
21. Zhang L, Zhang J. Impacts of Leisure and Tourism on the Elderly's Quality of Life in Intimacy: A Comparative Study in Japan. Sustainability. 2018;10:4861. Doi: 10.3390/su10124861.

22. Fong ZH, Tan S, Mahendran R, Kua EH, Chee T. Arts-based interventions to improve cognition in older persons with mild cognitive impairment: A systematic review of randomized controlled trials. Aging \& Mental Health. 2021; 25 (9): 1605-1617. doi: 10.1080 / 13607863.2020.1786802

23. Teh J, Tey N. Effects of selected leisure activities on preventing loneliness among older Chinese. SSM - Population Health. 2019;9:100479. doi: 10.1016 / j.ssmph.2019.100479.

24. Ihle A, Bavelier D, Maurer J, Oris M, Kliegel M. Internet use in old age predicts smaller cognitive decline only in men. Scientific Reports. 2020;10:8969. doi: 10.1038 / s41598-020-65846-9.

25. Howarth M, Brettle A, Hardman M, Maden M. What is the evidence for the impact of gardens and gardening on health and well-being: a scoping review and evidence-based logic model to guide healthcare strategy decision making on the use of gardening approaches as a social prescription. BMJ Open. 2020;10:e03692. doi: 10.1136/bmjopen-2020-036923.

26. Petretto D, Pili R. Ageing and COVID-19: What Is the Role for Elderly People?. Geriatrics. 2020;5(2):25. doi: 10.3390 / geriatrics5020025.

27. Airola E, Rasi P, Outila M. Older people as users and non-users of a video conferencing service for promoting social connectedness and well-being - a case study from Finnish Lapland. Educational Gerontology. 2020; 46(5):258-269. DOI: 10.1080 / 03601277.2020 .1743008

28. Paiva J, Andrade R, de Oliverira P, Duarte P, Santos I, Evangelista A, Theophilo R, de Andrade L, de H.C.Barreto I. Mobile applications for elderly healthcare: A systematic mapping. PLoS ONE. 2020;15(7):e0236091. Doi:10.1371/journal.pone.0236091

29. Ortega G, Rodríguez J, Maurer L, Witt E, Perez N, Reich A, Bates D. Telemedicine, COVID-19, and disparities: Policy implications. Health Policy and Technology. 2020;9:368-371. doi: 10.1016 / j.hlpt.2020.08.001.

30. Lam K, Lu A, Shi Y, Covinsky K. Assessing Telemedicine Unreadiness Among Older Adults in the United States during the COVID-19 Pandemic. JAMA Internal Medicine. 2020. doi: 10.1001 / jamainternmed.2020.2671.

31. Goodman-Casanova J, Dura-Perez E, Guzaman-Parra J, CuestaVargas A, Mayoral-Cleries F. Telehealth Home Support During COVID19 Confinement for Community-Dwelling Older Adults With Mild Cognitive Impairment or Mild Dementia: Survey Study. Med Internet Res. 2020;22(5):e19434. doi: 10.2196/ 19434.

32. Cimpeman M, Makovec M, Trkman P, Stanonik L. Older Adults' Perceptions of Home Telehealth Services. TELEMEDICINE and eHEALTH. 2020;19(10):786-90. doi: 10.1089/tmj.2012.0272.

33. Harrington R, Gray M, Jani A. Digitally enabled social prescriptions: adaptive interventions to promote health in children and young people. Journal of the Royal Society of Medicine. 2020;113(7):270-273. doi: 10.1177 / 0141076819890548.

34. Houston D, Aitalieva N, Morelock A, Shults C. Citizen Trust in Civil Servants: A Cross-National Examination. International Journal of Public Administration. 2016;39(14):1203-1214. DOI: 10.1080 / 01900692.2016 .1156696

35. Delany-Crowe T, Popay J, Lawless A, Baum F, MacDougall C, Eyk H, Williams $C$. The role of trust in joined-up government activities: Experiences from Health in All Policies in South Australia. Aust J Publ Admin. 2019:78(2):172-190. DOI:10.1111/1467-8500.12383

36. Sabat I, Neuman-Nöhme S, Elsem N, Pita P, Brouwer W, Exel V, Schreyögg J, Stargardt T. United but divided: Policy responses and people's perceptions in theEU during the COVID-19 outbreak. Health Policy. 2020;124:909-918.

37. Elgar F, Stefaniak A, Wohl M. The trouble with trust: Time-series analysis of social capital, income inequality, and COVID-19 deaths in 84 countries. Social Science \& Medicine. 2020;263:113386. doi: $10.1016 /$ j.socscimed.2020.113365.

38. Heliwell J. Determinants of Well-Being and Their Implications for Health Care. Ann Nutr Metab. 2019;74(suppl 2):8-14. doi: 10.1159/000499141.

39. Yu CY, Hou SI, Miller J. Health for Older Adults: The Role of Social Capital and Leisure-Time Physical Activity by Living Arrangements. Journal of Physical Activity \& Health. 2017;15(2):150-158. doi: 10.1123 / jpah.2017-0006.

40. Roth AN. Políticas públicas: formulación, implementación y evaluación. 1era edición. Bogotá: Ediciones Aurora;2009. p .73-105. 